\title{
Research on the Developmental Trend of Wearable Devices from the Perspective of Human-Computer Interaction
}

\author{
Tuanbu Wang ${ }^{1, a}$ and Wenzhun Huang ${ }^{1, b}$ \\ ${ }^{1}$ Xijing University, Xi'an, Shaanxi, China \\ a597669319@qq.com, ${ }^{b} 1051917312 @ q q . c o m$
}

Keywords: Wearable Devices; Human-Computer Interaction; Trend; Development; Perspective

\begin{abstract}
In this paper, we conduct research on the developmental trend of modern wearable devices from perspective of the human-computer interaction. Wearable computers have to face the primary products market, the development momentum is very fierce, can be applied to various fields, has a great market potential, as therefore the research of the technology is also more and more. Wearable computer as representative one kind of emerging scientific and technological achievements, to instill the advanced concept of "people-oriented", although is only in the initial stage, convenient but it easy to carry, etc. has been the favor of people, visible in the future after it has potential of technological progress. Our research integrates the human-computer interaction to enhance the traditional wearable devices that will optimize the performance.
\end{abstract}

\section{Introduction}

Intelligent is the inevitable trend in the development of science and technology, people's life will be changed with the development of the intelligent technology. In recent years, smart phone, smart TV industry rapid development, has become an indispensable product of people's lives. Is not only a mobile phone and television since the Google launched the first generation of GoogleGlass intelligent wearable device market had begun to obtain the sprouts [1-2].

Because wearable device is convenient to carry, good interactivity as has become a hot new product electronic equipment industry, military, commercial market with enormous potential. The primary characteristics of wearable computer could be organized as the follows. (1) Observability. Under the premise of wearable computing in the user agrees to, can be user continuous attention enough, and the user can keep the sustainable output media. (2) Controllability. Wearable computing at any time any place that can be to run the automation of operation, and the user can choose human intervention or disconnect as able to independently control circuit. (3) The exclusive. Wearable computers and other virtual reality technology, it will not cut off cut off the outside world, the application of wearable computer is at the same time, also can go need to focus on the other things, will not result in a delay to each other. (4) Non-restrictive. Use a wearable computer users, will not cause by is limited by basic geographical information calculation incompetent, whether in roaming, mobile, or walking around the application of wearable computing that can guarantee the value of its application, and does not interfere with people do other things [3-4].

Intelligent interactive technology need speech recognition technology and the general fusion of the basic brain-computer interface technology, the technology to improve the user experience of wearable devices as the goal, and the information exchange between the wearer and equipment to form a system of whole technology. A wide variety of wearable devices produced a lot of a wide variety of data. The data is large, high-speed, diversity, valuable, and that is precisely the basic characteristics of big data. Therefore, wearable device is doomed to be inseparable with big data and wearable devices if we want to further development that also must make full use of the data processing and the core visualization technology to provide new attraction, further enhance the user viscosity.

People use information system to complete the task in the process of a variety of basic information technology as the support, the computer as the platform, in the network environment by human and computer to implement interactive. In narrow sense, mainly is the process of information exchange 
between human and computer. Interaction is both have certain intelligence and information system of the cognitive system, their interaction process is a continuous and complex and dynamic process, the form of both sides of the general intellectual efficiency. Information system interaction efficiency is a collaborative efficiency, not a single person or the efficiency of the information system the higher the better, but the mutual coordination and information system, make the whole process of interaction to achieve the best efficiency with efficient and quick to complete the task [5].

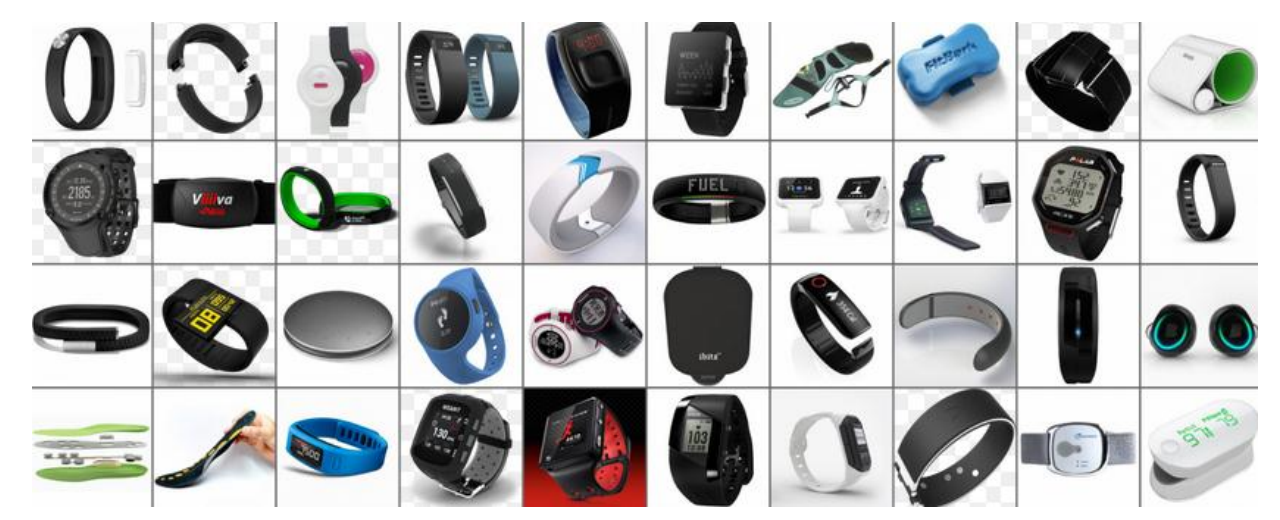

Figure 1. The Contemporary Wearable Devices

In this article, we conduct research on developmental trend of wearable devices from perspective of human-computer interaction. At present, wearable computer is still in primary stage of development, can usually be interpreted as another kind of new way of using computers. With related technology matures, wearable computer meter is used the external form and the using mode has changed a lot. In the following sub-sections, we will propose our suggestions and countermeasures.

\section{The Novel Methodology and Perspective}

The Human-Computer Interaction. Information quality is a measure of information system output. Information can be used by the quality of the following measures: the information output format and information output accuracy, timeliness, reliability, integrity, transparency of information output, etc.

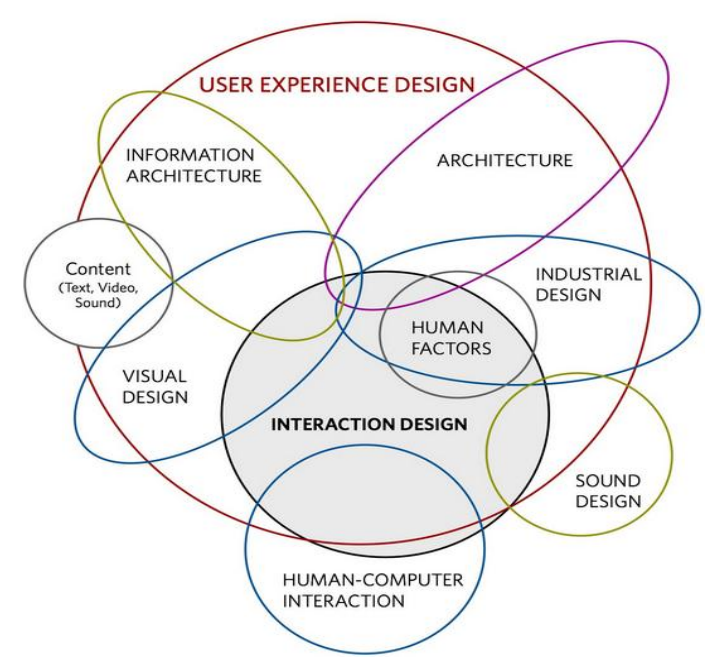

Figure 2. The Human-Computer Interaction Principles 
- According to the classification data acquisition processing mode. External processing type wearable devices, it is using wireless communication technology, with the general help of the blue-tooth interface, such as the access to external data and carries on the processing, cloud computing is a diversified approach one of the representative technologies.

- According to the application type classification. Wearable equipment categories of the general application of each are not identical, can be summed up in daily model and target model. Daily type that is daily application as the main purpose, that mainly including the Google glass mentioned in the article, Apple iWatch wrist strap and sports, etc.

- According to the feature classification. Feature sets forming of wearable devices will move the original equipment with integrated functions, to achieve a number of different functions of the traditional equipment, on the basis of extensions, such as apple just released iWatch products is to integrate the function of the smart phone on smart watches, more convenient.

The man-machine combination as low in order to better help users have more intelligent with the help of the method, must have a more advanced way of interaction. Increasing reality above refers to the real environment to provide information to cover, superposition of basic image, word, sound and hypertext in real environment, one of which remind, tips, mnemonic, annotation and interpretation and some other auxiliary functions and methodologies.

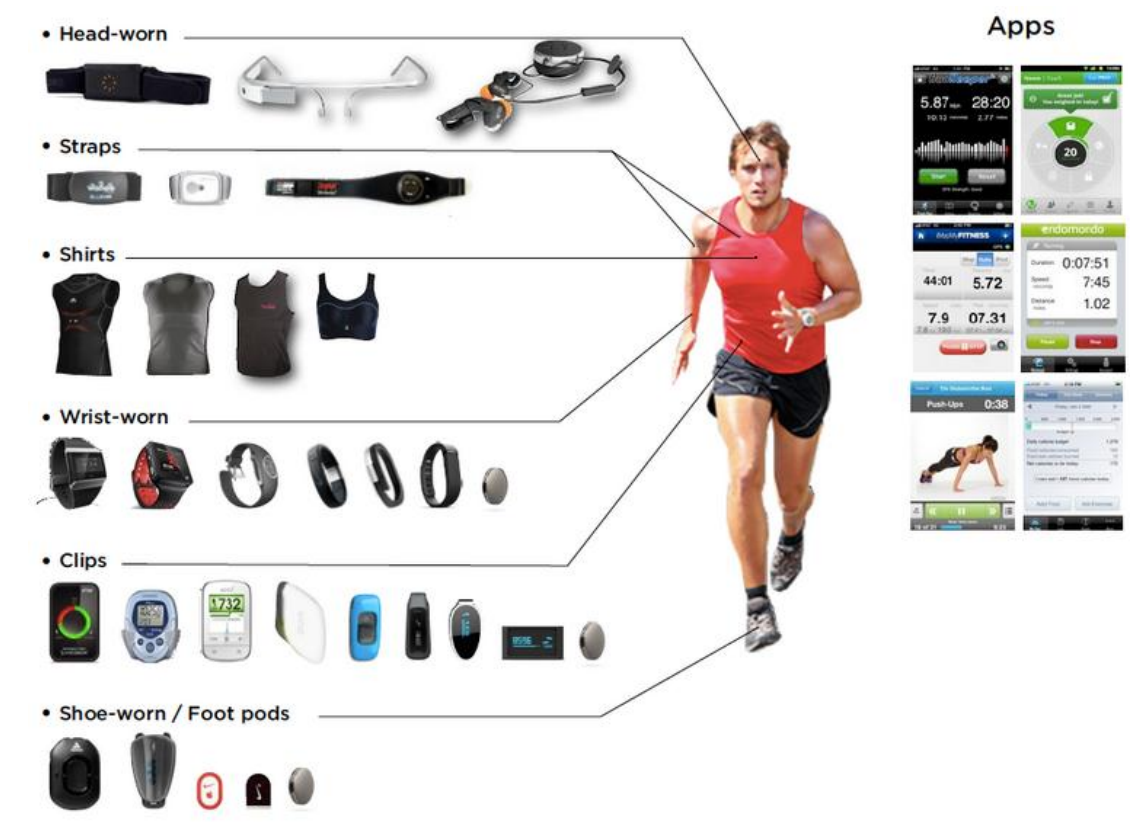

Figure 3. The Architecture and General Components of the Wearable Devices

The Augmented Reality. Augmented reality is reality and the general virtual environment in real time. Implementation of augmented reality is the key to data from the real world and virtual data in real-time and efficient integration of the world. AR's greatness lies in the computer, network, and the users are brought back to the real world. It generated by the computer system does not exist in the practical environment of virtual information, these virtual information can be use in a variety of ways such as vision, hearing, touch, smell sense, become a part of the real surroundings.

In order to achieve augmented reality, ARToolKit is a powerful tool for creating augmented reality application. To achieve any visual augmented reality, must be a computer with a camera. But even though we can use the ARToolKit, that the augmented reality application potential has not been fully 
excavated, one of the biggest reason is that users must display can contain the camera data, and can contain data from the computer processor running augmented reality application.

Solve the virtual objects and the real scene in three-dimensional space position of the consistency problem, that is, on the space integration. Because of general augmented reality technology has the characteristics of bricks and real-time interaction, augmented reality in the real world and virtual world integration research emphasizes the real world and virtual world. 3D registration technology through the actual object coordinates system the transformation of the relationship between the virtual objects into real scenario correctly that could be organized as follows [8].

- ARML: augmented reality markup language, is a kind of specification, allowing developers to create in a variety of mobile AR browsers display content.

- OMA: a standard organization, its aim is to seek a kind of has nothing to do with the system, open, make all kinds of mobile applications and services to worldwide standards of various kinds of terminal to realize the connectivity.

- The MPEG-7: a series of standards, known as basic multimedia content description interface standards. For all kinds of multimedia information to provide a standardized description, this kind of description and content it has a high correlation, convenient for users to better use of the multimedia information.

- WebGL: a 3D drawing standard, the drawing technology standards allow the JavaScript and OpenGL ES 2.0 together, by increasing the OpenGL ES 2.0 JavaScript binding, WebGL for the HTML 5 Canvas to provide $3 \mathrm{~d}$ hardware accelerated rendering.

The Brain Wave Control for Wearable Devices. Eeg is a kind of bioelectricity phenomenon, is the brain when the activity, the cortex cells formed between potential difference. Based on electrical wear equipment development has become the development trend of the future, and can effectively extract the characteristic value of brain waves, it objectively index is the main challenge of brainwave wear equipment development [9-10].

The main body of the mind control system by the brain module, blue-tooth module and the main control calculator of three parts, including module is responsible for the acquisition of human brain waves, brain waves is the only signal capture module. Brain wave module and data communication between master computers implemented by the blue-tooth module as master control computer is the core of the mind control system, it implements the analysis of brain wave data modeling and control instruction is given. For the need for human control object, the control of the motion control system based on the mind control can be liberating people's hands, for ordinary people can make the control more entertaining and at the same time also can let liberation hands engaged in other operations.

Based on Eeg brain-computer interface technology because of its academic value and general broad application prospect, become a hot research at home and abroad. Its application fields cover control prosthetic device, communication, military applications, video games and robot control, etc., it is not only a new way of assistive and control measures, can help to improve the quality of life of disabled persons, and for a normal person, can also provide a special information communication and basic entertainment. Computer scientists in the United States use brain waves control, key technology is the size of a ballpoint pen head nerve electrode, can directly into the brain and nerves. The electrode is connected to the embedded in the scalp of miniature amplifier and filter or otherwise a micro power supply is responsible for send signals to a computer near. The computer can make the quadriplegic disabled on the computer to perform some previous still belong to impossible. Receive brain wave computer installed the special software, and revised the general application software, used to collect and analyze data with control peripheral devices. 


\section{Conclusion and Summary}

In this paper, we conduct research on developmental trend of wearable devices from perspective of the human-computer interaction. Hybrid tracking technology plays very important role in the field of augmented reality. No of hybrid tracking technology, augmented reality will have to be limited in the limited space within the scope of application that can be sure that there will be more in the current development of novel hybrid tracking method, the calibration method and the time synchronization method is applied to track mixture, the mixture of advanced tracking technology will help augment reality involved in people's daily life, which may change the form of people's lives. In the recent and further future, we will verify the feasibility with the hardware implementation.

\section{References}

[1] Patel, Mitesh S., David A. Asch, and Kevin G. Volpp. "Wearable devices as facilitators, not drivers, of health behavior change." Jama 313.5 (2015): 459-460.

[2] Molina-Markham, Andres, et al. "Amulet: a secure architecture for mHealth applications for low-power wearable devices." Proceedings of the 1st Workshop on Mobile Medical Applications. ACM, 2014.

[3] Rettberg, Jill Walker. Seeing ourselves through technology: How we use selfies, blogs and wearable devices to see and shape ourselves. Palgrave Macmillan, 2014.

[4] Pyattaev, Alexander, et al. "Communication challenges in high-density deployments of wearable wireless devices." Wireless Communications, IEEE 22.1 (2015): 12-18.

[5] Radhakrishnan, Srinivasan, Arjun Duvvuru, and Sagar V. Kamarthi. "Investigating Discrete Event Simulation Method to Assess the Effectiveness of Wearable Health Monitoring Devices." Procedia Economics and Finance 11 (2014): 838-856.

[6] Jiang, Zhi Hao, et al. "A compact, low-profile metasurface-enabled antenna for wearable medical body-area network devices." Antennas and Propagation, IEEE Transactions on 62.8 (2014): 4021-4030. (In Chinese)

[7] Bito, Jo, and Manos M. Tentzeris. "A novel flexible wearable magnetic energy harvester utilizing inkjet masking techniques." Antennas and Propagation \& USNC/URSI National Radio Science Meeting, 2015 IEEE International Symposium on. IEEE, 2015.

[8] Leonov, Vladimir. "Thermoelectric energy harvesting of human body heat for wearable sensors." Sensors Journal, IEEE 13.6 (2013): 2284-2291.

[9] Lee, Jaehong, et al. "Conductive Fiber-Based Ultrasensitive Textile Pressure Sensor for Wearable Electronics." Advanced Materials 27.15 (2015): 2433-2439.

[10] Agoulmine, Nazim, Priyadip Ray, and T-H. Wu. "Efficient and cost-effective communications in ubiquitous healthcare: wireless sensors, devices and solutions [Guest Editorial]." Communications Magazine, IEEE 50.5 (2012): 90-91. 